\title{
Application of Artificial Intelligence in the Diagnosis and Treatment of COVID-19 Lung Disease
}

\author{
Khanjani $\mathrm{K}^{1 *}$, Mehralian S ${ }^{1}$, Jalaeian Zaferani $\mathrm{E}^{1}$, Teshnehlab $\mathbf{M}^{1}$, Nadji $\mathrm{A}^{2}$ and Pourabdollah $\mathbf{M}^{3}$ \\ ${ }^{1}$ Intelligent Systems Lab, Electrical \& Computer Eng. Faculty of K. N. Toosi University of Technology, Tehran, Iran \\ ${ }^{2}$ Virology Research Center, NRITLD, Masih Daneshvari Hospital, Shahid Beheshti University of Medical Sciences, Daarabad, Niavaran, Tehran 19569- \\ 44413, Iran \\ ${ }^{3}$ Department of Pathology, Chronic Respiratory Diseases Research Center, National Research Institute of Tuberculosis and Lung Diseases (NRITLD), \\ Shahid Beheshti University of Medical Sciences, Tehran, Iran
}

^Correspondingauthor: K. Khanjani, Intelligent Systems Lab, Electrical \& Computer Eng. Faculty of KN Toosi University of Technology, Tehran, Iran; Email: kaviankhanjani@ email.kntu.ac.ir

Received: November 20, 2020; Accepted: November 26, 2020; Published: December 01, 2020

\section{Introduction}

COVID-19 disease spread worldwide in 2019 from Wuhan, China, for unknown reasons $[1,2]$. The disease was caused by the SARS-CoV-2 virus and infected many people in different parts of the world in a short time. According to global statistics, about 255 million people have been infected with the illness so far, and 5.12 million people have died from it [3]. Emergencies have led health professionals and centers to develop guidelines to prevent the transmission of the sickness chain and to treat people. Common symptoms include fever, cough, tiredness, and loss of smell or taste. In addition, patients may experience sore throat, headache, irritated eyes, and diarrhea. More severe cases of the disease can include chest pain and shortness of breath which the person should go to a doctor and hospital immediately [4]. Since its inception, the virus has shown mutations that have led to increased transmission and severity of the disease, which has ultimately increased mortality. Some obvious variants are Alpha, Beta, Gamma, and Delta species which the Delta mutation has become more intense. Although the process of universal vaccination has made humans more resistant to the virus, the disease is now leading to injury and death [5].

\section{Methods of Diagnosis}

One of the most important things to deal with COVID-19 is the process of its diagnosis. So that diagnosis in the early stages of the conflict plays an important role in the treatment of patients. A variety of methods have been used for this purpose, the most common of which is the RT-PCR test [6]. Another commonly used solution is to use medical CT scans and X-rays, which doctors can use to identify areas of lung involvement and severity [7]. The significant problem with this method is that pulmonary symptoms do not usually occur in the early stages of the illness. Therefore, this method leads to delays in the treatment process of patients [8]. Among other procedures, the diagnostic approach is based on blood tests, which are further discussed in this study. Performing this test has advantages over other methods, including the cost of performing it compared to other ways is less and the result is available in a shorter time [9]. Combining artificial intelligence techniques with diagnostic tests can significantly increase the accuracy and speed of detection [10]. For example, deep convolutional neural networks can categorize and segment medical images based on symptoms and infections in the lungs [11] or analyze and categorize medical and statistical data using various machine learning methods and multilayer perceptron networks [12].

\section{Data Information}

The data used in this study include 1104 cases, of which 531 samples are related to persons with COVID-19 and 573 samples are related to people who do not have this disease. The blood parameters used include CRP, Lymphocyte, Platelet, W.B.C, and LDH. The target label corresponding to each blood test sample includes zero or one, which distinguishes between the two categories. Data and labels have been prepared and provided by specialists and physicians of Masih Daneshvari hospital located in Iran.

\section{Details of Experiment}

The mechanism used in the classifier design is the K-Fold criterion $(\mathrm{K}=5)$. Thus, at each stage, $80 \%$ of the data is used to train and design the categorizer and $20 \%$ to test it. The above method repeats the training process five times and the final results are obtained based on the average of these steps. Various methods were implemented on blood test data, and the best result was obtained by the ensemble approach, which is the result of combining the three methods of K-nearest-neighborhood, random forest, and multilayer perceptron. This method determines the final class by voting between the three mentioned categories. K-nearest-neighborhood and random forest methods are common machine learning methods that are available in (13)and (14), respectively. Information about the multilayer perceptron network is also available in (15).

\section{Results}

The classification results are based on the confusion matrix and the criteria including accuracy, precision, recall, and F1-score which 
Figure 1: Confusion matrices of train (left-side) and test (right side).

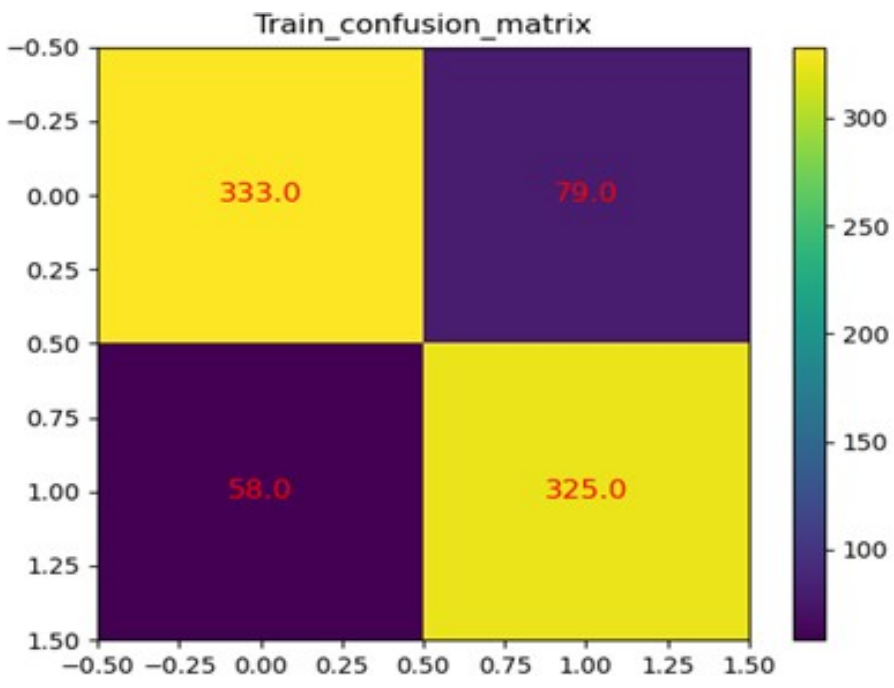

are represented in 1 to 4 equations. 'Negative' corresponds to people who do not have COVID-19 and Positive is related to ones with this disease.

$$
\begin{gathered}
\text { True Negative }+ \text { True Positive } \\
\begin{array}{c}
\text { Accuracy }= \\
\text { True Negative }+ \text { True Positive }+ \text { False Positive }+ \text { False Negative }
\end{array} \\
\text { Recall }=\begin{array}{c}
\text { True Positive } \\
\text { True Positive }+ \text { False Negative }
\end{array} \\
\text { Precision }=\begin{array}{c}
\text { True Positive } \\
\text { True Positive }+ \text { False Positive }
\end{array} \\
\begin{array}{c}
2 * \text { Recall } * \text { Precision }) \\
\text { Recall }+ \text { Precision }
\end{array}
\end{gathered}
$$

The Confusion matrices of Trian and test are represented in Figure 1. Element 11 of matrices belong to the number of non-COVID persons that are classified correctly and element 22 is related to the number of COVID-19 patients who have been correctly diagnosed.

\section{Conclusion}

According to the results, only several blood parameters can be used to successfully diagnose COVID-19 disease with $82.7 \%$ accuracy, $84.9 \%$ recall, $80.4 \%$ precision, and $82.6 \%$ F1-score, on average. The more valid data available, the higher the percentages of accuracy and sensitivity. On the other hand, due to the diversity of artificial intelligence structures, the performance of the classifier can be improved with appropriate changes. This method can be used by the public at a lower cost in areas that do not have sufficient facilities.

\section{References}

1. Chaolin Huang, Yeming Wang, Xingwang Li, Lili Ren, et al. (2020) Clinical features of patients infected with 2019 novel coronavirus in Wuhan, China. The Lancet Journal 497-506.

2. Wu, F., Zhao, S., Yu, B, Chen YM, Wang W, et al. (2020) A new coronavirus associated with human respiratory disease in China. Nature 580: 7. [crossref]

3. Ritchie H, Mathieu E, Rodés-Guirao L, Appel C, et al. (2020) Coronavirus Pandemic (COVID-19). Our World in Data.

4. Singhal T (2020) A Review of Coronavirus Disease. The Indian Journal of Pediatrics 1-6.

5. Forchette L, William S, Liu T (2021) A comprehensive review of COVID-19 virology, vaccines, variants, and therapeutics., Curr Med Sci 9: 1-15. [crossref]

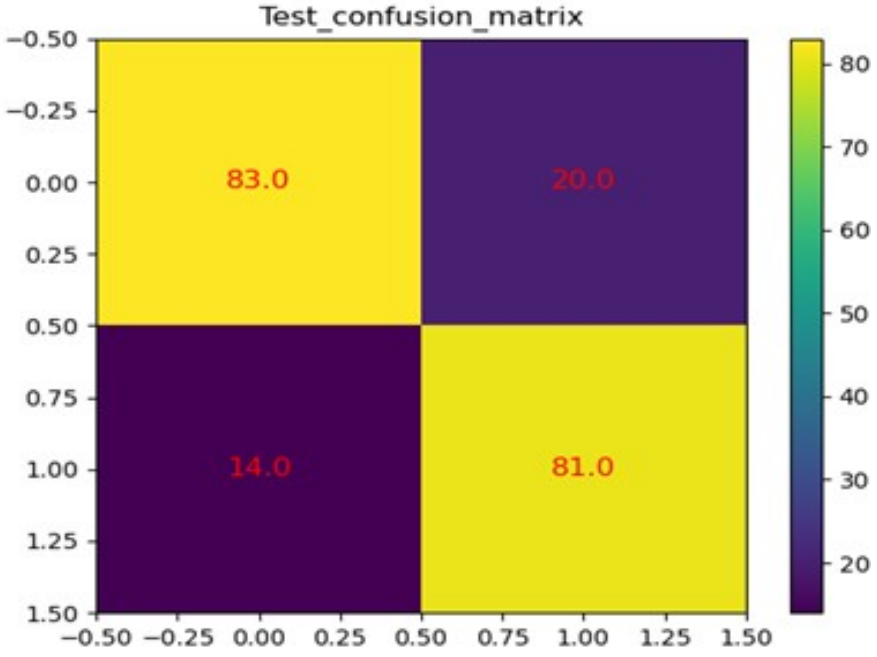

6. Kasteren PBV, Veer BVD, Brink SVD, Wijsman L, Jong JD, et al. (2020) Comparison of seven commercial RT-PCR diagnostic kits for COVID-19. Clinical Virology 128: 104412. [crossref]

7. Shah FM, Joy SKS, Ahmed F, Hossain T, Humaira M, et al. (2021) A Comprehensive Survey of COVID-19 Detection Using Medical Images. SN comput Sci 2: 434 [crossref]

8. Bernheim A, Mei X, Huang M, Yang Y, Fayad ZA, et al. (2020) Chest CT Findings in Coronavirus Disease-19 (COVID-19): Relationship to Duration of Infection. Radiology 295: 200463.

9. Mehralian S, Zaferani J, Effat, Shahrzad S, Kashefinishabouri Farnaz, et al. (2021) Rapid COVID-19 Screening Based on the Blood Test using Artificial Intelligence Methods. Journal of Control 14

10. Hussain AA, Bouachir O, Turjman FA, Aloqaily M (2020) AI techniques for COVID-19. IEEE Access 8: 128776-128795.

11. Rahman T, Khandakar A, Qiblawey Y, Tahir A, Kiranyaz S, et al. (2021) Exploring the effect of image enhancement techniques on COVID-19 detection using chest X-ray images. Computers in Biology and Medicine 132: 104319. [crossref]

12. Borghi, PH, Zakordonets O, Teixeira JP (2021) A COVID-19 time series forecasting model based on MLP ANN. Procedia Computer Science 181: 940-947. [crossref]

13. Theodoridis, Konstantinos KS (2009) Pattern Recognition. Fourth 56-59.

14. Breiman L (2001) Random forests. Machine learning 45: 5-32.

15. Mohammad Teshnehlab, Pourya Jafari (2015) Neural Networks and Advanced Neuro-Controllers. Tehran: KN Toosi University of Technology.

\section{Citation:}

Khanjani K, Mehralian S, Jalaeian Zaferani E, Teshnehlab M, Nadji A, et al. (2021) Application of Artificial Intelligence in the Diagnosis and Treatment of COVID-19 Lung Disease. J Int Pers COVID-19 Volume 1(2): 1-2. 